\title{
Preserved tactile acuity in older pianists
}

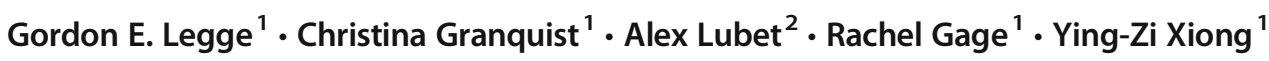

Published online: 13 August 2019

(C) The Psychonomic Society, Inc. 2019

\begin{abstract}
A previous study from our lab demonstrated retention of high tactile acuity throughout the lifespan in blind subjects in contrast to the typical decline found for sighted subjects (Legge, Madison, Vaughn, Cheong \& Miller, Percept Psychophys, 70 (8), 1471-1488, 2008). We hypothesize that preserved tactile acuity in old age is due to lifelong experience with focused attention to touch and not to blindness per se. Proficient pianists devote attention to touch - fingerings and dynamics - over years of practice. To test our hypothesis, we measured tactile acuity in groups of ten young (mean age 24.5 years) and 11 old (mean age 64.7 years) normally sighted pianists and compared their results to the blind and sighted subjects in our 2008 study. The pianists, like the subjects in 2008, were tested on two tactile-acuity charts requiring active touch, one composed of embossed Landolt rings and the other composed of dot patterns similar to braille. For both tests, the pianists performed more like the blind subjects than the sighted subjects from our 2008 study. For the ring chart, there was no significant difference in tactile acuity between the young and old pianists and no significant difference between the pianists and the blind subjects. For the dot chart, the pianists showed an age-related decline in tactile acuity, but not as severe as the sighted subjects from 2008. Our results are consistent with the hypothesis that lifelong experience with focused attention to touch acts to preserve tactile acuity into old age for both blind and sighted subjects.
\end{abstract}

Keywords Tactile acuity $\cdot$ Aging $\cdot$ Blind $\cdot$ Pianist

\section{Introduction}

Several studies have found that blind subjects have better tactile acuity than age-matched sighted controls (Goldreich \& Kanics, 2003, 2006; Stevens, Foulke \& Patterson, 1996; Van Boven, Hamilton, Kauffman, Keenan, \& Pascual-Leone, 2000). Blind subjects can resolve gaps between two dots that are roughly $15 \%$ smaller than sighted subjects (summarized by Legge et al., 2008, Table 1). Empirical evidence indicates that the superior tactile acuity of blind subjects is due to extensive tactile experience rather than to deprivation of visual input (Wong, Gnanakumaran, \& Goldreich, 2011a).

Electronic supplementary material The online version of this article (https://doi.org/10.3758/s13414-019-01844-y) contains supplementary material, which is available to authorized users.

Gordon E. Legge

legge@umn.edu

1 Department of Psychology, University of Minnesota, 75 East River Road, Minneapolis 55455, MN, USA

2 School of Music, University of Minnesota, 2106 4th St S, Minneapolis 55455, MN, USA
Both blind and sighted subjects display an age-related decline in tactile acuity when measured with gap separation or grating orientation. Threshold gap size on the fingertip increases about $1 \%$ per year resulting in nearly a doubling in tactile thresholds from 20 to 80 years (Stevens \& Choo, 1996).

Braille characters are composed of dot patterns whose gap separation is close to the acuity limit. The age-related decline in tactile acuity should make braille reading more difficult in old age. However, there is no compelling evidence that older braille readers have difficulty reading. Previously, we addressed this discrepancy by describing two new measures of tactile acuity and tests of blind and sighted subjects across a wide age range (Legge et al., 2008).

Braille reading requires moving the fingertip across dot patterns. Prior tests of tactile acuity have usually employed passive stimulation, that is, presentation of a tactile stimulus to an immobile finger. Passive tests of tactile acuity may yield different information to that from active tests.

We designed two tactile-acuity charts requiring active movement, possibly more closely related to braille reading and other tactile activities of daily life. The dot chart (Fig. 1, right) has symbols similar to braille. Subjects move their fingers across the symbols. In our 2008 study (Exp. 1), we measured tactile acuity on the dot chart for 49 blind subjects 
Table 1 Comparison of the groups of pianists in the current study with the groups of blind and sighted controls in Legge et al. (2008, Experiment 2)

\begin{tabular}{|c|c|c|c|c|c|c|}
\hline \multirow[t]{2}{*}{ Groups } & \multicolumn{2}{|l|}{ Pianist Subjects } & \multicolumn{2}{|c|}{$\begin{array}{l}\text { Blind Subjects } \\
\text { (2008 Study Experiment 2) }\end{array}$} & \multicolumn{2}{|c|}{$\begin{array}{l}\text { Control Subjects } \\
\text { (2008 Study Experiment 2) }\end{array}$} \\
\hline & $\begin{array}{l}\text { Young } \\
(\mathrm{n}=10)\end{array}$ & $\begin{array}{l}\text { Old } \\
(\mathrm{n}=11)\end{array}$ & $\begin{array}{l}\text { Young } \\
(\mathrm{n}=10)\end{array}$ & $\begin{array}{l}\text { Old } \\
(n=13)\end{array}$ & Young $(\mathrm{n}=10)$ & $\begin{array}{l}\text { Old } \\
(n=10)\end{array}$ \\
\hline $\begin{array}{l}\text { Gender } \\
(\mathrm{F} / \mathrm{M})\end{array}$ & $6 / 4$ & $10 / 1$ & $6 / 4$ & $9 / 4$ & $6 / 4$ & $5 / 5$ \\
\hline Age, y (range) & $24.5 \pm 4.0(19-30)$ & $64.7 \pm 6.3(57-75)$ & $31.2 \pm 5.8(23-39)$ & $67.4 \pm 8.7(56-81)$ & $29.3 \pm 4.8(22-35)$ & $70.3 \pm 9.5(57-85)$ \\
\hline
\end{tabular}

Age values are given as mean $\pm \mathrm{SD}$

ranging in age from 18 to 74 years, and 83 sighted subjects aged from 12 to 82 years. The sighted subjects exhibited the age-related decline in tactile acuity found with passive stimulation. Surprisingly, the blind subjects showed no age-related decline.

Because our blind subjects were all braille readers and familiar with the test symbols on the dot chart, we designed a second chart for comparing tactile acuity in the two groups. Our second chart (Fig. 1, left) uses embossed landolt rings. The four symbols are defined by the orientation of the gap in the ring, and are likely to be of roughly equal salience for blind and sighted subjects. In our 2008 study (Exp. 2), we compared the tactile acuity of blind and sighted subjects in young and old groups (Table 1). Once again, we found that sighted subjects demonstrated the typical age-related decline in tactile acuity while the young and old blind subjects did not differ in their tactile acuity.

The novel finding from our 2008 study was that the blind subjects retain high tactile acuity across the lifespan, in contrast to the age-related decline of tactile acuity for sighted subjects.
Why is there this difference between sighted and blind subjects? A lifetime of intensive tactile experience might protect against age-related decline of tactile acuity for the blind subjects. If so, would sighted subjects who regularly devote more attention to tactile function also exhibit preserved tactile acuity into old age? Proficient pianists devote attention to touch - fingerings and dynamics -over years of practice. Ragert et al. (2004) have already shown that proficient pianists exhibit better tactile acuity on their index fingers than nonmusicians. We decided to compare tactile acuity for young and old pianists.

\section{Methods}

\section{Subjects}

We measured tactile acuity in groups of young and old sighted pianists. We compared their performance with data from corresponding groups of blind and sighted subjects in
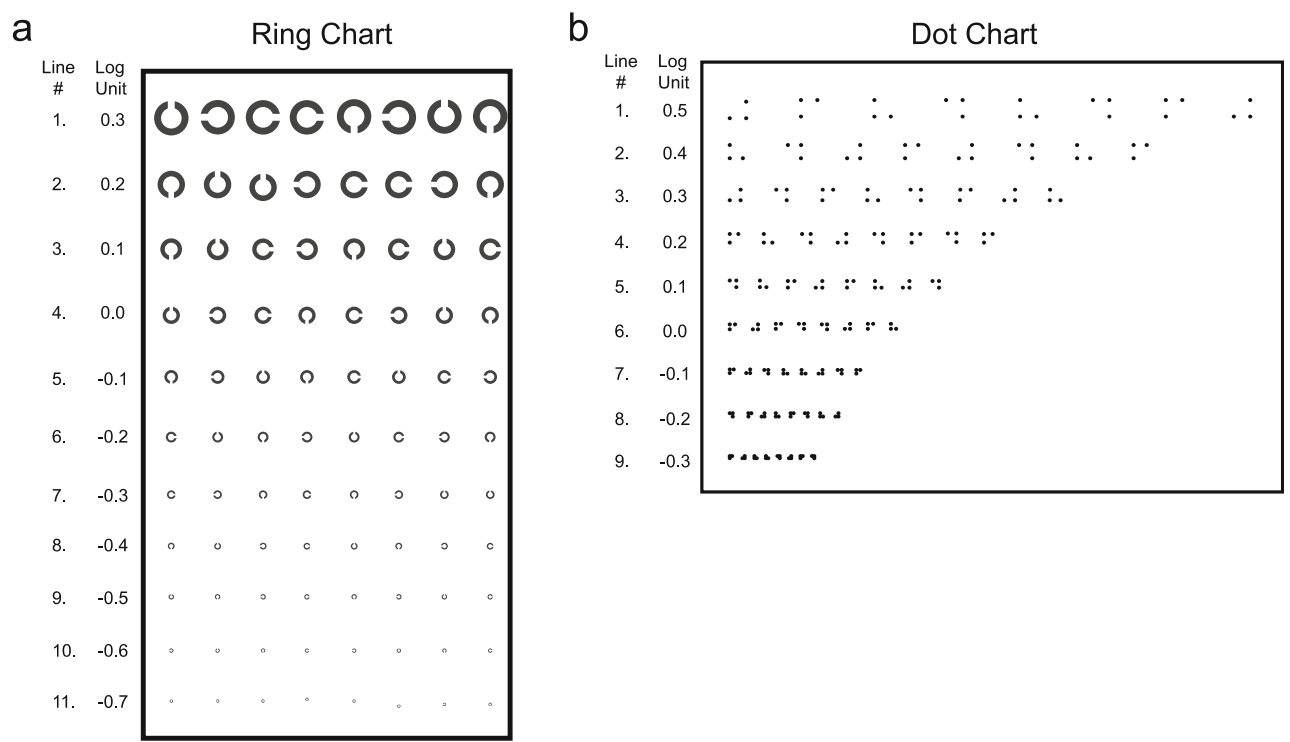

Fig. 1 Illustration of the ring chart (a) and the dot chart (b). Both charts are composed of four symbols, arranged in rows of decreasing size in 0.1 log-unit steps ( $26 \%$ change in size). Line 4 on the ring chart and line 6 on the dot chart are the reference lines $(0.0 \log$ units) 
Experiment 2 of our 2008 paper. For clarity, we refer to the sighted subjects from 2008 as controls.

We planned on a sample size of ten per group based on our 2008 study. Specifically, a sample size of nine to 12 in each group was sufficient to show a significant interaction between age group and vision status, with an effect size $\left(\eta p^{2}\right)$ of 0.18 . A prior analysis showed that to achieve the same effect size, at a 0.05 significance level with a power of $80 \%$, a sample size of ten per group is required.

Recruiting criteria included age from 18 to 30 years or 55 to 75 years, self-reported corrected-to-normal vision, healthy hands and fingers, and a history of many years of dedicated piano playing. The subjects completed the Edinburgh Handedness Inventory, a hand-health questionnaire and a piano experience questionnaire. All subjects gave informed consent, and the study protocol was approved by the University of Minnesota's Institutional Review Board.

There were ten young pianists (mean age 24.5 years; six female and four male; all right-handed) and 11 old pianists (mean age 64.7 years; ten female and one male; ten righthanded and one left-handed). Table 1 compares the age and gender of the young and old pianists in the current study with the blind subjects and controls from 2008.

Most of the young pianists were current or former students from the University of Minnesota School of Music. Most had been playing the piano since early childhood with the number of years of continuous playing ranging from 6 to 24 (mean = 16.8 years). Most reported substantial practice ranging from $2.19 \mathrm{~h}$ per week to $42 \mathrm{~h}$ per week $($ mean $=16.5 \mathrm{~h})$. Many of the older subjects were piano or school music teachers. Most of them had also been playing since childhood, with the number of years of playing ranging from 35 to 68 years (mean 56.4 years). The older subjects reported somewhat less practice than the younger subjects, ranging from $1 \mathrm{~h}$ per week to $20 \mathrm{~h}$ per week $($ mean $=5.3 \mathrm{~h})$.

\section{Test charts}

The dot and ring charts were custom-built for our 2008 study. For construction details, see Legge et al. (2008). Bruns et al. (2014) described practical methods for producing such charts and demonstrated that test-retest reliability was better than with passive tests of tactile acuity.

The four test symbols on the dot chart (Fig. 1, right) are the braille letters ( $\mathrm{d}, \mathrm{f}, \mathrm{h}$, and $\mathrm{j}$ ), all with three dots. The symbols differ only in the location of the missing fourth dot in the square. Blind braille readers name the letters, and sighted subjects indicate location of the missing dot, e.g., "upper right." The chart has nine lines of eight characters: dot separations decreased by $0.1 \log$ unit ( $26 \%$ ) per line. The dot spacing on the sixth line, labeled $0.0 \log$ units, corresponds to standard braille characters (center-to-center spacing of $2.28 \mathrm{~mm}$ ).
The ring chart (Fig. 1, left) also has eight symbols per line, decreasing in size in 0.1 log-unit steps. The 0.0 line on the ring chart has a gap size of $2.40 \mathrm{~mm}$, similar to the dot spacing on the 0.0 line of the dot chart. All subjects indicated the location of the gap for each ring symbol as "top," "bottom," "left" or "right."

Both tests were scored on a logarithmic scale following standard methods for scoring visual acuity on a symbol-bysymbol basis (Ferris et al., 1982). Specifically, each line corresponds to a score of $0.1 \log$ unit, and each symbol $1 / 8$ of one line or $0.0125 \log$ units. The total score was obtained by the following formula: acuity $=$ smallest line attempted (log unit) + total number of errors $\times 0.0125$. A smaller score (and more negative values) indicates better tactile acuity. For example, an acuity of 0.0 means that the subject could identify symbols on the 0.0 line but not smaller symbols, and an acuity of -0.1 means that the subject could identify symbols on the line one step smaller than the 0.0 line.

\section{Procedure}

Pianists were blindfolded and tested on the index finger of their dominant hand. The charts were placed on a table in front of the subject. The subject read the row containing the largest symbols first, and proceeded line by line to the smallest symbols.

The subjects were tested twice on each chart, with half of them tested in the order dot-ring-dot-ring and the other half in the order ring-dot-ring-dot. The tests were not timed, and accuracy was stressed over speed. The session lasted 1-2 h.

\section{Results}

\section{Relationship between tactile acuity and age}

Figures 2 and 3 compare the tactile acuities of the young and old Pianist groups from the present study with the Blind and Control groups from Experiment 2 in our 2008 study. The bars in Fig. 2 show average group data. The data points in Fig. 3 show tactile acuity as a function of age for individual subjects. In both figures, the left vertical scale is tactile acuity in log units. The right vertical scale shows corresponding tactile acuity values in $\mathrm{mm}$ (dot spacing or gap size).

We begin by summarizing the main findings. Comparing the old groups on the ring chart (Fig. 2a), the Pianist group was very similar to the 2008 Blind group, and both groups had better tactile acuity than the 2008 Controls. Comparing the old groups on the dot chart (Fig. 2b), the Blind group had better tactile acuity than the Pianist group, and the Pianist group had better tactile acuity than the Controls.

The individual data and regression lines in Fig. 3 indicate that age had no significant effect on tactile acuity for the blind subjects (both charts) and the pianists (ring chart). There was an effect of age on the dot chart for the pianists but the effect 


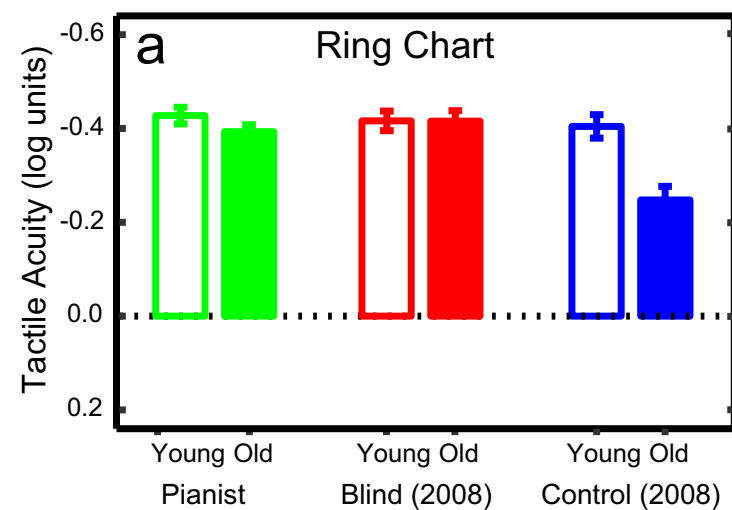

Fig. 2 Average tactile acuities for groups of young and old pianists (this study) and blind and control subjects from Legge et al. (2008). Values are shown for the ring chart (a) and the dot chart (b). Acuity values refer to

was weaker than for the controls. Age affected tactile acuity for the controls on both charts.

Statistical analysis follows We performed linear mixed-effects modeling on the tactile acuity with chart types (ring and dot), gender (female, male), age group (young, old), and vision status (Pianist, Blind, Control) as fixed factors (Pinheiro \& Bates, 2000). Random effects included intercepts and slopes for each chart and each subject. The normality of residuals was confirmed by a diagnostic plot (normal q-q plot) and a Kolmogorov-Smirnov test $(p>0.05)$. There were significant main effects of chart $(F(1,52)=591.10, p<0.001)$, age group $(\mathrm{F}(1,52)=25.78, \mathrm{p}<0.001)$, and vision status $(\mathrm{F}(2,52)=$ $24.89, \mathrm{p}<0.001)$. There were also significant interactions between chart and age group $(\mathrm{F}(1,52)=4.13, \mathrm{p}=0.047)$,

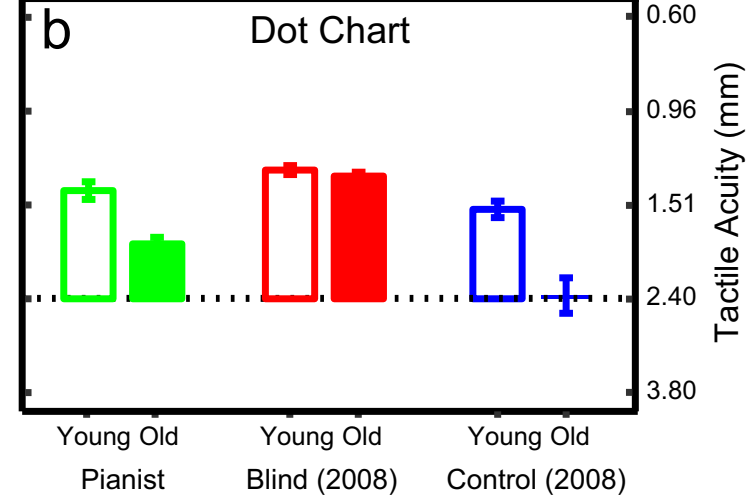

the gap size in the Landolt rings, or to the center-to-center spacing of adjacent dots, expressed in log units (left scale) and $\mathrm{mm}$ (right scale). Error bars represent \pm 1 standard error

between chart and vision status $(\mathrm{F}(2,52)=12.35, \mathrm{p}<0.001)$, and between age group and vision status $(\mathrm{F}(2,52)=8.84, \mathrm{p}<$ $0.001)$. Gender showed no significant main effect $(F(1,52)=$ $2.25, \mathrm{p}=0.14$ ) or interaction with other variables (all $\mathrm{p}>$ 0.1 ). Post hoc analyses were then performed with Bonferroni correction, with findings as follows.

Ring chart There was no difference between the three young groups $(p=1.00)$. For the old subjects, the tactile acuity of the Control group was significantly worse than both the Blind ( $\mathrm{p}$ $<0.001)$ and Pianist groups $(\mathrm{p}=0.004)$, and there was no difference between the Blind and Pianist groups $(p=1.00)$. The young and old groups showed no significant age effect in the Blind $(\mathrm{p}=0.98)$ and Pianist $(\mathrm{p}=0.48)$ groups, while there was a significant effect in the Control group $(p<0.001)$.
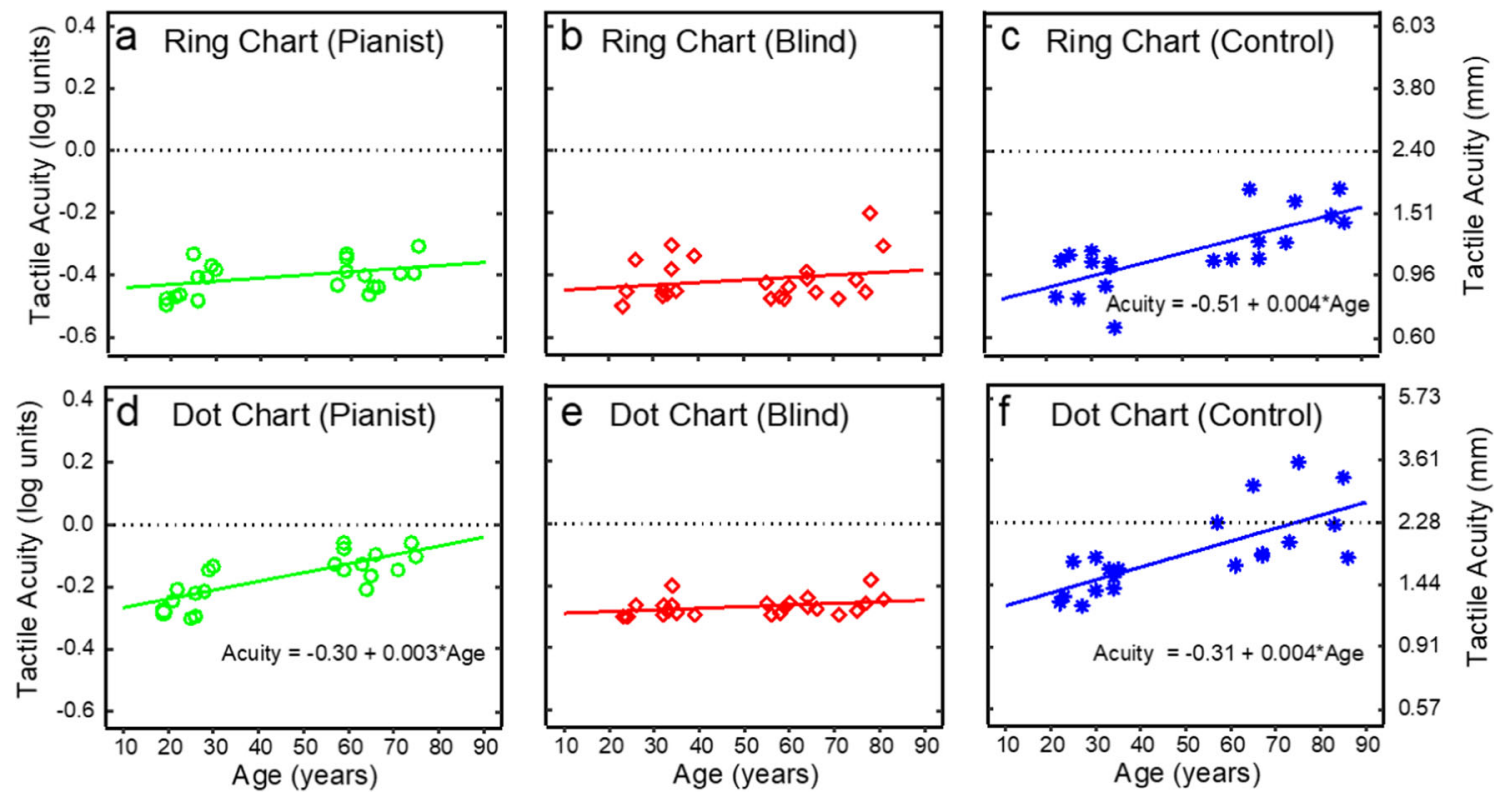

Fig. 3 Tactile acuities measured with the ring chart $(\mathbf{a}, \mathbf{b}, \mathbf{c})$ and the dot chart $(\mathbf{d}, \mathbf{e}, \mathbf{f})$ are plotted as a function of age. Individual values are shown squares regression lines are shown for each group. Equations are shown for regression lines with significant effects of age 
Dot chart For the young groups, there was no difference between Pianist versus Blind $(p=0.55)$ or Pianist versus Control $(p=0.71)$ groups, but there was a significant difference between Blind and Control $(p=0.042)$ groups. For the old groups, the Pianist group was significantly worse than the Blind group ( $\mathrm{p}=0.007)$, but significantly better than the Control group $(p=0.018)$. Comparing young and old groups, significant age effects were found in both Control $(p<0.001)$ and Pianist $(\mathrm{p}=0.023)$ groups, but not in the Blind group $(\mathrm{p}=$ 0.76).

An important result is the significant interaction between aging and experience. While tactile acuity did not differ significantly between the groups of young pianists and controls, the old pianists' mean acuity was $0.11 \log$ units $(29 \%)$ better on the dot chart and $0.18 \log$ units $(51 \%)$ better on the ring chart than the controls.

Figure 3 shows individual tactile acuities (means of two tests) as a function of age for the three groups. For the ring chart, regression analysis indicated that age was a significant predictor only for the control subjects $\left(\mathrm{p}<0.001\right.$, adjusted $\mathrm{R}^{2}$ $=0.53$ ). For the dot chart, age was a significant predictor for both the Pianist group $\left(\mathrm{p}<0.001\right.$, adjusted $\left.\mathrm{R}^{2}=0.58\right)$ and the Control group $\left(\mathrm{p}<0.001\right.$, adjusted $\left.\mathrm{R}^{2}=0.52\right)$.

\section{Relationship between tactile acuity and piano experience}

Two items related to piano experience were included in a questionnaire filled out by our 21 pianists: number of years playing piano, and hours of weekly practice. Both of these variables were confounded with age: old pianists had a longer history of playing, and devoted less weekly time to practice (see Subjects section). To clarify, we constructed linear regression models with acuity as the dependent variable, and age, years of playing, and weekly practice time as independent variables. For acuity on the ring chart, the best model (adjusted $\mathrm{R}^{2}=0.37$ ) showed that only weekly practice time was a predictor (beta $=-0.003, p=0.002$ ). For acuity on the dot chart, the best model (adjusted $\mathrm{R}^{2}=0.58$ ) showed that only age was a predictor $($ beta $=0.003, \mathrm{p}<0.001$ ).

\section{Discussion}

The central issue addressed in this paper is whether pianists with normal vision show preserved tactile acuity in old age. Do they behave like blind subjects or do they show the decline in tactile acuity typical of most sighted subjects? The qualified answer is that they behave more like blind subjects, demonstrating better tactile acuity in old age than sighted controls.

Results from the ring chart showed no significant differences between the pianists and the blind groups. For the dot chart, the pianists did demonstrate an age-related decline in tactile acuity, but less severe than that for the sighted controls.

What might account for the difference between the two charts? The dot chart requires recognition of the spatial arrangement of three dots and may be a more demanding task. This extra task demand may have uncovered a performance difference between old pianists and old blind subjects not evident on the ring chart. Because the dot patterns corresponded to braille characters, and our blind subjects were all braille readers, their better performance may also be related to familiarity with the dot patterns.

Most of our subject groups (Table 1) had more females than males. Might the gender distributions have affected our group comparisons? It is known that average tactile acuity, measured with passive grating-orientation discrimination, is better for females than males (Peters et al., 2009; Wong et al., 2011b). The difference is related to the smaller finger size in females and possibly to a corresponding higher density of Merkel receptors in the fingertip (Peters et al., 2009). Kalisch et al. (2012) found the opposite gender effect in an active haptic object-recognition task; males performed better than females. As discussed in the Results section, we did not find a significant gender effect in our groups, nor in our 2008 study. To further explore this issue, we reanalyzed the data from the present study, excluding male subjects. This reduced sample, composed of females only, yielded the same general pattern of results (see the Supplementary Material), that is, preserved tactile acuity in old age for the pianists and better tactile acuity for old pianists than for sighted controls. It is possible that the acuity advantage exhibited by females in the grating-orientation threshold task exists because this task is primarily dependent on sensoryreceptor sampling at the fingertip. The active-acuity measured in our study may be more reliant on higher-level cortical limitations, which may not exhibit gender differences.

Some other studies have considered the interacting effect of experience and age on tactile acuity. Guest et al. (2014) tested normally sighted adults in the age range $18-58$ years. The subjects provided self-reported assessments of tactile experience. Tactile acuity was measured with a grating-orientation test. They found an age-related decline but no relationship with tactile experience. The authors acknowledged that finding effects of tactile experience might require testing groups with exceptional tactile expertise.

Reuter et al. (2012) tested groups of young, middle-aged, and older subjects divided into novices and experts based on occupation. The occupations included those requiring manual dexterity, such as opticians, goldsmiths, dentists, and watchmakers. The subjects were tested on several tactile and haptic tasks. The results showed an age-related decline in several of the tasks, including grating-orientation discrimination, but no difference between experts and novices. In a subsequent study, Reuter et al. (2014) measured evoked potentials in response to tactile stimulation and also accuracy on tactile 
discrimination tasks. Both the evoked potentials and the behavioral results revealed differences based on expertise and aging. They concluded that tactile expertise results in improved perceptual performance but that both experts and non-experts demonstrate the same age-related decline.

Kerr et al. (2008) measured grating-orientation thresholds for a group of experienced tai chi practitioners. Tai chi requires focused attention on body extremities including the finger tips, but does not involve high-acuity tasks. The experienced practitioners had better tactile acuity than age and gender-matched controls. Kerr et al. also found a trend toward reduced impact of age on the tactile acuity of their older practitioners.

Why should playing the piano result in better tactile acuity? Piano performance does not require the fine spatial pattern resolution tested by our charts. Repetitive tapping of keys on a regular basis, such as computer keyboarding, does not seem to be an explanation. Tremblay et al. (2002) compared tactile acuity (grating-orientation discrimination) for frequent computer users ( $>2 \mathrm{~h}$ per day) and occasional or non-computer users $(<$ $2 \mathrm{~h}$ per day). Tactile thresholds were actually poorer in the frequent users, although the effect was only significant for the female subjects. In their study of tai chi practitioners, Kerr et al. (2008) proposed that focused attention on sensory and haptic functioning of the fingers promotes enhanced tactile acuity.

Why might focused tactile experience preserve tactile acuity into old age? Several studies have pointed to the association between haptic performance and cognition in understanding the impact of age on haptic object recognition (Kalisch et al., 2012) and spatial updating by touch (Giudice et al., 2017). Dinse et al. (2010) argued that the more complex the haptic or tactile task, the greater the involvement of central (cortical) mechanisms and the greater the impact of training. Certainly, both braille reading and piano performance involve high-level cognitive function as well as early sensory function. It is possible that focused tactile experience over a lifetime results in cortical plasticity that offsets or compensates for declining sensory acuity in the fingertips. Tactile acuity, measured with a task requiring active touch, may be more reflective of the involvement of higher-level cortical function in tactile perception than passive tests.

What might be the neural basis for the superior tactile acuity of pianists compared with sighted controls? Wong et al. (2011a) reviewed two forms of cortical plasticity potentially relevant to experience-dependent improvement in tactile acuity - intra-modal and cross-modal plasticity. Intra-modal plasticity refers to changes in sensorimotor cortex due to tactile experience as demonstrated in the expanded representations of the fingers of the left hand of normally sighted string players (Elbert et al., 1995) and the braille-reading fingers of blind subjects (Sterr et al., 1998). Cross-modal plasticity refers to recruitment of visual cortex in response to intensive tactile input as demonstrated for blind braille readers (Sadato et al., 1996) in low vision (Cheung et al., 2009) and even sighted subjects following 5 days of visual deprivation (Merabet et al., 2008). Both mechanisms may participate in the retention of high tactile acuity throughout the lifespan in blind subjects. It seems likely that the primary mechanism for superior tactile acuity in sighted pianists is intra-modal plasticity associated with modified representations of fingers or hands in sensorimotor cortex. A reviewer mentioned the intriguing possibility that the better acuity of older blind subjects than pianists for the braille-like symbols on the dot chart, but not the ring chart, might be due to a benefit from cross-modal plasticity associated with braille reading.

If attention plays a role in either type of experiencedependent cortical plasticity, we would expect to find evidence for attentional modulation of neural response in sensory cortex. Brain-imaging studies have shown that attention modulates retinotopically specific regions of primary visual cortex V1 (Gandhi, Heeger \& Boynton, 1999; Martinez et al., 1999). High-field fMRI has also shown attentional modulation of sensorimotor cortex S1 specific to the fingertips (Puckett et al., 2017). It is possible that recurring and extensive attentional modulation of sensorimotor cortex over a lifetime could modify the sensory representation of the fingers in pianists.

Finally, we found evidence for a relationship between the amount of weekly piano practice and tactile acuity (data from the ring chart), but not the number of years of piano playing. Ragert et al. (2004) also reported a significant correlation between tactile acuity and the amount of daily practice among their proficient pianists, but no significant correlation with the number of years playing. Wong et al. (2011a) reported a correlation between grating-orientation thresholds on the fingertip of braille readers and weekly reading time. Collectively, these results may indicate that a high level of persisting intensive tactile experience can offset deficits in tactile acuity due to age.

Our results are consistent with the hypothesis that lifelong experience with focused attention to touch may act to preserve tactile acuity into old age for both blind and sighted subjects.

Acknowledgements This research was supported by National Institutes of Health Grant EY002934 and a grant from the Helen Keller Foundation.

Open Practices Statement

The data for all experiments are available at the Data Repository of the University of Minnesota (https://doi.org/10.13020/fj2x-vk94). None of the data or materials for the experiment reported here was preregistered.

\section{References}

Bruns, P., Camargo, C. J., Campanella, H., Esteve, J., Dinse, H. R., \& Roder, B. (2014). Tactile acuity charts: a reliable measure of spatial acuity. PLoS One, 9(2), e87384.

Cheung, S. H., Fang, F., He, S., \& Legge, G. E. (2009) Retinotopically specific reorganization of visual cortex for tactile pattern recognition. Curr Biol, 19(7):596-601.

Dinse, H. R., Tegenthoff, M., Heinisch, C., \& Kalisch, T. (2010). Ageing and Touch. In E. B. Goldstein (Ed.), Encyclopedia of perception (Vol. 1, pp. 21-24). Thousand Oaks, CA: Sage. 
Elbert, T., Pantev, C., Wienbruch, C., Rockstroh, B. \& Taub, E. (1995). Increased cortical representation of the fingers of the left hand in string players. Science 270, 305-307.

Ferris, F. L., 3rd, Kassoff, A., Bresnick, G. H., \& Bailey, I. (1982). New visual acuity charts for clinical research. Am J Ophthalmol, 94(1), 91-96.

Gandhi, S. P., Heeger, D. J., \& Boynton, G. M. (1999). Spatial attention affects brain activity in human primary visual cortex. Proceedings of the National Academy of Sciences of the USA, 96(6), 3314-3319.

Giudice, N. A., Bennett, C. R., Klatzky, R. L., \& Loomis, J. M. (2017). Spatial updating of haptic arrays across the life span. Experimental Aging Research, 43(3), 274-290. doi:https://doi.org/10.1080/ 0361073X.2017.1298958

Goldreich, D., \& Kanics, I. M. (2003). Tactile acuity is enhanced in blindness. J Neurosci, 23(8), 3439-3445.

Goldreich, D., \& Kanics, I. M. (2006). Performance of blind and sighted humans on a tactile grating detection task. Percept Psychophys, 68(8), 1363-1371.

Guest, S., Mehrabyan, A., Ackerley, R., \& McGlone, F. (2014). Tactile experience does not ameliorate age-related reductions in sensory function. Experimental Aging Research, 40, 81-106.

Kalisch, T., Kattenstroth, J. C., Kowalewski, R., Tegenthoff, M., \& Dinse, H. R. (2012). Cognitive and tactile factors affecting human haptic performance in later life. PLoS One, 7(1), e30420.

Kerr, C. E., Shaw, J. R., Wasserman, R. H., Chen, V. W., Kanojia, A., Bayer, T., \& Kelley, J. M. (2008). Tactile acuity in experienced Tai Chi practitioners: evidence for use dependent plasticity as an effect of sensory-attentional training. Exp Brain Res, 188(2), 317-322.

Legge, G. E., Madison, C., Vaughn, B. N., Cheong, A. M., \& Miller, J. C. (2008). Retention of high tactile acuity throughout the life span in blindness. Percept Psychophys, 70(8), 1471-1488.

Martinez, A., Anllo-Vento, L., Sereno, M. I., Frank, L. R., Buxton, R. B., \& Dubowitz, D. J., et al. (1999). Involvement of striate and extrastriate visual cortical areas in spatial attention. Nature Neuroscience, 2, 364-369.

Merabet, L. B., et al. (2008). Rapid and reversible recruitment of early visual cortex for touch. PLoS One 3(8): e3046.

Peters, R. M., Hackeman, E., \& Goldreich, D. (2009). Diminutive digits discern delicate details: fingertip size and the sex difference in tactile spatial acuity. J Neurosci 29:15756-15761.

Pinheiro, J., \& Bates, D. (2000). Mixed-Effects Models in S and S-PLUS: Springer.

Puckett, A. M., Bollmann, S., Barth, M., \& Cunnington, R. (2017). Measuring the effects of attention to individual fingertips in somatosensory cortex using ultra-high field (7T) fMRI. Neuroimage, 161, 179-187. doi:https://doi.org/10.1016/j. neuroimage.2017.08.014

Ragert, P., Schmidt, A., Altenmuller, E., \& Dinse, H. R. (2004). Superior tactile performance and learning in professional pianists: evidence for meta-plasticity in musicians. Eur J Neurosci, 19(2), 473-478.

Reuter, E.-M., Voelcker-Rehage, C., Vieluf, S., \& Godde, B. (2012). Touch perception throughout working life: effects of age and expertise. Experimental Brain Research, 216(2), 287-297.

Reuter, E.-M., Voelcker-Rehage, C., Vieluf, S., Winneke, A. H., \& Godde, B. (2014). Extensive occupational finger use delays age effects in tactile perception - an ERP study. Attention, Perception, \& Psychophysics, 76(4), 1160-1175.

Sadato, N., Pascual-Leone, A., Grafman, J., Ibanez, V., Deiber, M.P., Dold, G., \& Hallett, M. (1996). Activation of the primary visual cortex by Braille reading in blind subjects. Nature 380, 526-528.

Sterr, A., Muller, M. M., Elbert, T., Rockstroh, B., Pantev, C., \& Taub, E. (1998). Perceptual correlates of changes in cortical representation of fingers in blind multifinger Braille readers. J Neurosci, 18(11), 4417-4423.

Stevens, J. C., \& Choo, K. K. (1996). Spatial acuity of the body surface over the life span. Somatosensory \& motor research, 13(2), 153-166. doi:https://doi.org/10.3109/08990229609051403

Stevens, J. C., Foulke, E., \& Patterson, M. Q. (1996). Tactile acuity, aging, and braille reading in long-term blindness. Journal of Experimental Psychology: Applied, 2(2), 91-106. doi:https://doi. org/10.1037/1076-898X.2.2.91

Tremblay, F., Mireault, A. C., Letourneau, J., Pierrat, A., \& Bourrassa, S. (2002) Tactile perception and manual dexterity in computer users. Somatosens Mot Res, 19:101- 108.

Van Boven, R. W., Hamilton, R. H., Kauffman, T., Keenan, J. P., \& Pascual-Leone, A. (2000). Tactile spatial resolution in blind Braille readers. Neurology, 54(12), 2230-2236. doi:https://doi.org/ 10.1212/wnl.54.12.2230

Wong, M., Gnanakumaran, V., \& Goldreich, D. (2011a). Tactile spatial acuity enhancement in blindness: evidence for experiencedependent mechanisms. J Neurosci, 31(19), 7028-7037.

Wong, M., Hackeman, E., Hurd, C., \& Goldreich, D. (2011b) Short-term visual deprivation does not enhance passive tactile spatial acuity. PLoS One, 6:e25277.

Publisher's note Springer Nature remains neutral with regard to jurisdictional claims in published maps and institutional affiliations. 